\title{
Variants of theorems of Schur, Baer and Hall
}

\author{
B. A. F. Wehrfritz ${ }^{1}$
}

Received: 24 March 2016 / Published online: 27 June 2017

(C) The Author(s) 2017. This article is an open access publication

\begin{abstract}
If a group $G$ is 'restricted' modulo its hypercentre, then to what extent does $G$ have an equally restricted normal subgroup $L$ with $G / L$ hypercentral? We consider these questions where restricted means finite- $\pi$, Chernikov, locally finite- $\pi$, polycyclic or polycyclic-by-finite.
\end{abstract}

Keywords Hypercentre $\cdot$ Hypocentre $\cdot$ Central series

Mathematics Subject Classification 20F14 · 20E15

\section{Introduction}

For any group $G$ denote its centre by $\zeta_{1}(G)$ and its hypercentre by $\zeta(G)$. If $t$ is a positive integer, say $t=\Pi_{\text {primes }} p^{e(p)}$, let $e(t)$ denote the maximum of the $e(p)$ (so $e(1)=0)$ and $h(t)$ the sum of all the $e(p)$. Set $a(t)=[e(t) / 2]+1$, where $[r]$ denotes the integer part of a real number $r$, and set $b(t)=t^{(e(t)+1) / 2}$. Obviously $b(t) \leq t^{a(t)}$. The following variant of theorems of Schur and Baer was essentially proved by de Falco et al. [2].

Theorem A (cf. [2,5]) Let $G$ be a group with $G / \zeta(G)$ finite of order $t$. Then $G$ has a normal subgroup $L$ with $G / L$ hypercentral and with $L$ of finite order dividing $t^{a(t)+1}$ and at most $b(t) t$.

Communicated by F. de Giovanni.

$凶 \quad$ B. A. F. Wehrfritz

b.a.f.wehrfritz@qmul.ac.uk

1 Queen Mary University of London, London E1 4NS, UK 
de Falco et al. [2] gives no specific bounds. The later paper [5] by Kurdachenko et al. contains two proofs of Theorem A, one shorter with no bounds and one with just a bound for $|L|$ slightly larger than $b(t) t$.

There are variants of the classical Schur and Baer theorems where finite is replaced by notions like Chernikov, polycyclic or locally finite, see [7], especially Page 115. Here we consider corresponding questions in the context of Theorem A. The following is the main result of this paper.

Theorem B Let $G$ be a group with $G / \zeta(G)$ a Chernikov group. Then $G$ has a normal Chernikov subgroup $L$ with $G / L$ hypercentral.

A minor variation to our proof of Theorem B gives yet another short proof of Theorem A. In fact we prove Theorem A with rather better bounds than those stated above, but with bounds less briefly explained. Let $Z$ be a central subgroup of a group $G$ of finite index dividing $t$. Then (Schur's theorem) the order $\left|G^{\prime}\right|$ of the derived subgroup of $G$ is finite and in fact boundedly so (e.g. the easy proof of [11] 1.18, yields that $\log _{t}\left|G^{\prime}\right| \leq(t-1)^{2}+1$. Given $t$ define the integers $c(t)$ and $d(t)$ as follows: $c(t)$ is the least integer such that for any $G$ and $Z$ as above (but with fixed $t$ ), $\left|G^{\prime}\right|$ divides $t^{c(t)}$ and $d(t)$ is the least integer with $\left|G^{\prime}\right| \leq d(t)$. Notice that if $s$ divides $t$, then $c(s) \leq c(t)$ and $d(s) \leq d(t)$. By Theorem 1 of [12] we have $c(t) \leq[e(t) / 2]+1$ and $d(t) \leq \bar{t}^{(e(t)+1) / 2}$. Hence Theorem A follows from the following.

Theorem C Let $G$ be a group with $Z=\zeta(G)$ of finite index in $G$ dividing $t$. Then $G$ has a normal subgroup $L$ with $G / L$ hypercentral and with $L$ of finite order dividing $t^{c(t)+1}$ and at most $d(t) t$.

Wiegold [13] has a different type of bound for $d(t)$. Assume $t>1$, let $q$ be the least integer to divide $t$ and set $t^{\prime}=\log _{q} t$ and $t^{\prime \prime}=\left[t^{\prime}\right]$; clearly $e(t) \leq h(t) \leq t^{\prime \prime} \leq t^{\prime} \leq t$. Then Wiegold proves that $d(t) \leq t^{\left(t^{\prime}-1\right) / 2}$. In fact one can do a little better than this (see [12] Theorems 2 and 3), namely that $c(t) \leq\left[t^{\prime \prime} / 2\right]$ and $d(t) \leq t^{\left(t^{\prime \prime}-1\right) / 2}$ unless $t=p^{e} q$ or $p q^{e}$ with $p>q$ primes and $e \geq 1$ when $c(t) \leq\left[t^{\prime \prime} / 2\right]+1$. Further if $t=p q^{e}$ with $e \geq 2$ or if $t=p^{e} q$ with $p^{e}>q^{e+1}$, then $d(t) \leq t^{\left(t^{\prime \prime}-1\right) / 2}$. With the exceptional $t=\bar{p}^{e} q$ (e.g. $t=6$ ) we have of course Wiegold's bound $d(t) \leq t^{\left(t^{\prime}-1\right) / 2}$.

The obvious analogues of Theorem B, with Chernikov replaced by polycyclic or polycyclic-by-finite, are false, see Example 1 below. We do however have the following easier result.

Theorem D Let $G$ be a group with $G / \zeta(G)$ a locally finite $\pi$-group for some set $\pi$ of primes (e.g. $\pi$ the set of all primes). Then $G$ has a locally finite, normal $\pi$-subgroup $L$ with $G / L$ hypercentral.

Casolo, Dardano and Rinauro in their recent paper [1] prove the corresponding result to Theorem A in the context of Hall's theorem. Specifically they prove the following.

Theorem E (see [1] Theorem A) Let L be a finite normal subgroup of the group G such that $G / L$ is hypercentral. Then the index $(G: \zeta(G))$ is finite and divides $|A u t L| .\left|\zeta_{1}(L)\right|$. 
Simple examples show that the corresponding statements are false with finite replaced by Chernikov, polycyclic, polycyclic-by-finite, or locally finite, see Examples 2, 3 and 4 below. Theorem $\mathrm{E}$ is a very easy consequence of our final theorem.

Theorem $\mathbf{F}$ Let $A$ be a finite abelian normal subgroup of the group $G$ and let $H$ be a normal subgroup of $G$ in $C_{G}(A)$ and containing $A$. Suppose every finite image of $G / C_{G}(H)$ is nilpotent. Then $(H / A) \cap \zeta(G / A)=A(H \cap \zeta(G)) / A$; that is, if $\phi$ denotes the natural projection of $G$ onto $G / A$, then $H \phi \cap \zeta(G \phi)=(H \cap \zeta(G)) \phi$.

Whenever we have $A \leq K \leq H$ note that $(K / A) \cap \zeta(G / A)=A(K \cap \zeta(G)) / A$. To derive Theorem E from Theorem F, set $H=C_{G}(L)$ and $A=H \cap L$. Clearly $H / A$ is $G$-isomorphic to $H L / L \leq G / L$, which is hypercentral. Consequently $H / A \leq$ $\zeta(G / A)$. Also $L \leq C_{G}(H)$, so $G / C_{G}(H)$ is hypercentral. Then Theorem F implies that $H \leq A . \zeta(G)$. Clearly $(G: H)$ divides $|A u t L|$. Therefore $(G: \zeta(G))$ divides $\mid A$ ut L $|| A \mid$. .

\section{Proof of the Theorems}

Lemma 1 Let $V$ be a finite elementary abelian p-group and $G$ a nilpotent subgroup of Aut $V$. Then as $G$-module $V=V_{1} \oplus V_{2} \oplus \cdots \oplus V_{r}$, where for each $i$ the $G$ composition factors of $V_{i}$ are all $G$-isomorphic. In particular if $V$ as $G$-module has a non-trivial factor centralized by $G$, then $V$ has a non-zero element fixed by $G$ and $a$ non-trivial image centralized by $G$.

To obtain such a decomposition of $V$, see [10] 7.15. Note that the hypothesis there that the field $F$ is algebraically closed is only used to ensure that the Jordan decomposition of each $g$ in $G$ takes place in $G L(n, F)$. If $g \in G$ has finite order, then trivially $g_{u}$ and $g_{d}$ lie in $\langle g\rangle$, so here, as $H$ is finite, we can dispense with the algebraic closure hypothesis. (Actually it suffices just to have $F$ perfect, e.g. see [9] 3.1.6, which of course automatically covers the $F=G F(p)$ case.)

Remark Suppose $G$ is a nilpotent group and $V$ a finite $G$-module such that $V=$ $[V, G$ ]. If $q$ is prime, Lemma 1 shows that $V / q V$ has no trivial $G$-composition factors. Thus nor does any $G$-image of $V / q V$; in particular nor does any $q^{i} V / q^{i+1} V$. Applying this for every $q$ dividing the order of $V$ shows that $V$ itself has no trivial $G$-composition factors.

As well as $\zeta(G)=\cup_{w \geq 0} \zeta_{w}(G)$, the hypercentre of $G$ we consider $\gamma G=$ $\cap_{w \geq 0} \gamma^{w+1} G$, the hypocentre $G$; here $w$ runs over the ordinals, $\left\{\zeta_{w}(G)\right\}$ is the upper central series of $G$ and $\left\{\gamma^{w+1}(G)\right\}$ is the lower central series of $G$. Let $k \geq 0$ and $t \geq 1$ be integers. If $\left(G: \zeta_{k}(G)\right)=t$, then clearly $\zeta(G)=\zeta_{k+e(t)}(G)$. Also by Baer's theorem $\left|\gamma^{k+1} G\right|$ is finite (see [8] 14.5.1), so $G / \gamma G$ is nilpotent. Then $G / \gamma G \cdot \zeta_{k}(G)$ is nilpotent of order dividing $t$, so $G / \gamma G$ is nilpotent of class at most $k+e(t)$ and $\gamma G=\gamma^{k+e(t)+1} G$. Suppose instead that $\left|\gamma^{k+1} G\right|=d$. Clearly then $\gamma G=\gamma^{k+e(d)+1}(G)$. Also the upper central series of $G$ intersected with $\gamma^{k+1} G$ has length at most $e(d)$ and $\zeta(G) /\left(\zeta(G) \cap \gamma^{k+1} G\right)$ embeds into $G / \gamma^{k+1} G$ as a $G$-group and hence has $G$-central height at most $k$. Therefore $\zeta(G !)=\zeta_{k+e(d)}(G)$. The above 
might seem rather pedantic, but one needs to be slightly careful in dealing with $\gamma G$ for infinite groups $G$. We use these remarks below.

We now start on the proofs of Theorem B and, indirectly, Theorem $\mathrm{C}$. Thus below $G$ denotes a group with $G / \zeta(G)$ a Chernikov group. Set $Z=\zeta(G)$ and $\Gamma=\gamma G$.

Suppose first that $G$ is finite and that $(G: Z)$ divides $t$. Now $G / C_{G}(Z)$ stabilizes the upper central series of $G$ and hence is nilpotent. Therefore $\Gamma \leq C_{G}(Z), \Gamma \cap Z \leq$ $\zeta_{1}(\Gamma),(\Gamma: \Gamma \cap Z)$ divides $t$ and $\left|\Gamma^{\prime}\right|$ divides $t^{c(t)}$ and is at most $d(t)$. Set $V=\Gamma / \Gamma^{\prime}$. Clearly $\Gamma$ centralizes $V$, so $G / C_{G}(V)$ is nilpotent. By the Remarks above $V$ has no trivial $G$-composition factors, so $\Gamma \cap Z \leq \Gamma^{\prime}$. Thus $\left(\Gamma: \Gamma^{\prime}\right)$ divides $t$. Therefore $|\Gamma|$ divides $t^{c(t)+1}$ and is at most $d(t) t$.

Now suppose that $G$ is finitely generated. Again we have $t=(G: Z)$ finite. Also $G$ is polycyclic-by-finite, so there exists an integer $k$ with $\zeta_{k}(G)=Z$. By Baer's theorem $\gamma^{k+1} G$ is finite, so $\Gamma \leq \gamma^{k+1} G$ is finite and $G / \Gamma$ is nilpotent. Now $G$ is residually finite. Hence there is a normal subgroup $N$ of $G$ of finite index with $\Gamma \cap N=\langle 1\rangle$. Clearly $Z N / N \leq \zeta(G / N)$ and $\Gamma \cong \Gamma N / N=\gamma(G / N)$. By the finite case we have that $|\Gamma|$ divides $t^{c(t)+1}$ and is at most $d(t) t$. Also by the finite case we have $[\Gamma, Z] \leq \Gamma \cap N=\langle 1\rangle$ and $\Gamma \cap Z \leq \Gamma \cap \Gamma^{\prime} N=\Gamma^{\prime}$.

The Proof of Theorem B. Suppose $X \leq Y$ are finitely generated subgroups of $G$. Clearly $\gamma X \leq \gamma Y$, so $L=\cup_{X} \gamma X$ is a normal subgroup of $G$. By the finitely generated case above we have that $[\gamma X, X \cap Z]=\langle 1\rangle$ and $\gamma X \cap Z \leq(\gamma X)^{\prime}$; further $X / \gamma X$ is nilpotent. If $x \in L$ and $z \in Z$, there exists an $X$ with $x \in \gamma X$ and $z \in X \cap Z$. Then $[x, z]=1$ and hence $[L, Z]=\langle 1\rangle$. Also

$$
L \cap Z=\cup_{X}(\gamma X \cap Z) \leq \cup_{X}(\gamma X)^{\prime}=L^{\prime} .
$$

Now $G / L$ is locally nilpotent since each $X / \gamma X$ is nilpotent and locally nilpotent Chernikov groups are hypercentral. Hence $G / L Z$ and $G / L$ are hypercentral. Further $L /(L \cap Z)$ is Chernikov and $L \cap Z \leq \zeta_{1}(L)$. Therefore $L^{\prime}$ is Chernikov by Polovickii's theorem (see [7] 4.23). Consequently $L$ is Chernikov. The proof is complete.

The Proof of Theorem $C$. Here we have $(G: Z)$ dividing $t$. Let $X$ be a finitely generated subgroup of $G$ with $X Z=G$. By the finitely generated case we have that $X / \gamma X$ is nilpotent and that $|\gamma X|$ divides $t^{c(t)+1}$ and is at most $d(t) t$. Choose $X$ so that $|\gamma X|$ is maximal. If $Y$ is any finitely generated subgroup of $G$ containing $X$, then $\gamma X \leq \gamma Y$ since $[\gamma X, X]=\gamma X$. By the maximal choice of $X$ we have $\gamma X=\gamma Y$. This is for all such $Y$ and consequently $L=\gamma X$ is normal in $G$. If $\psi$ is the natural map of $G$ onto $G / L$, then $X \psi$ is nilpotent and $G \psi=X \psi \cdot Z \psi$. Consequently $G \psi$ is hypercentral. The proof is complete.

Comments on the above proofs. Notice that in general, unlike the finitely generated case, in Theorem $\mathrm{C}$ we cannot prove that $\gamma G$ is finite; just consider the infinite locally dihedral 2-group. However, since $L=\gamma X=[\gamma X, X]$, so $L=[L, G] \leq \gamma G$ and $G / \gamma G$ is hypercentral. Further $L$ is actually the hypercentral residual of $G$ and in particular $L$ is fully invariant in $G$.

A similar remark applies to Theorem B. If $A=\oplus_{i \geq 1}\left\langle a_{i}\right\rangle$ is free abelian of infinite rank and $x \in A u t A$ is given by $a_{i} x=a_{i-1}+a_{i}$ for all $i$ (with $a_{0}=0$ ), then the split 
extension $G$ of $A$ by $\langle x\rangle$ is hypercentral and yet $\gamma G=A$ is not Chernikov. Suppose $\alpha=\operatorname{ch}(G)$, the central height of $G$, and $\beta=\operatorname{ch}(G / L)$. Assuming $(G: Z)=t$, if $e=e(t)$, then $\beta \leq \alpha+e$. On the other hand if $|L|=d$ and if $f=e(d)$, then $\alpha \leq f+\beta$, so if either of $\alpha$ and $\beta$ is infinite, they both are and $\alpha \leq \beta \leq \alpha+e$.

Let $k \geq 0$ and $t \geq 1$ be integers. Suppose $G$ is a group with $\left(G: \zeta_{k}(G)\right)$ finite. By Baer's theorem $\gamma^{k+1} G$ is finite. More precisely there exists an integer-valued function $\tau(k, t)$ such that if $\left(G: \zeta_{k}(G)\right)$ divides $t$, then $\left|\gamma^{k+1} G\right|$ divides $t^{\tau(k, t)}$. For set $\tau(0, t)=1$ and (via Schur's theorem) set $\tau(1, t)=c(t)$. Suppose $k \geq 2$ and $\left|\gamma^{k}(G) . \zeta_{1}(G) / \zeta_{1}(G)\right|$ divides $s$. Then by [8] 14.5.2, or more precisely by its proof, $\left|\gamma^{k+1} G\right|$ divides $(s t)^{2 \tau(1, s t)+1}$. Thus we can define $\tau(k, t)$ inductively on $k$ by setting

$$
\tau(k, t)=(\tau(k-1, t)+1)(2 \tau(1, s t)+1) \text { for } s=t^{\tau(k-1, t)} .
$$

The above implies (cf. [1] Proposition 3) that if $\left(G: \zeta_{k}(G)\right)=t$, then the order of $\gamma^{2 k+1} G$ divides a power of $t$ whose exponent is bounded by a function of $t$ only, namely it divides $\max \left\{t^{c(t)+1}, t^{\tau(2 . c(t), t)}\right\}$. For as we saw above $G / \gamma G$ is nilpotent of class at most $k+e(t)$, so if $k \geq e(t)$, then $\left|\gamma^{2 k+1} G\right|=|\gamma G|$, which divides $t^{c(t)+1}$ and if $k \leq e(t)$, then $\left|\gamma^{2 k+1} G\right|$ divides $t^{\tau(2 . e(t), t)}$, since $\tau(k, t)$ is an increasing function of $k$.

Lemma 2 Let $G$ be a $\pi$-torsion-free group for $\pi$ some set of primes. If $G / \zeta(G)$ is a locally finite $\pi$-group, then $G=\zeta(G)$.

Proof If $X$ is a finitely generated subgroup of $G$, then $X$ is nilpotent-by-finite, $\zeta(X)=$ $\zeta_{k}(X)$ for some finite $k$ and $X / \zeta_{k}(X)$ is a finite $\pi$-group. Hence $\gamma^{k+1}(X)$ is also a finite $\pi$-group (e.g. [7] Page 115 or use the above). But $G$ is $\pi$-torsion-free; therefore $\gamma^{k+1}(X)=\langle 1\rangle$ and so $G$ is locally nilpotent. But then $\zeta(G)$ is $\pi$-isolated in $G$ (see [4] 4.8b). Therefore $\zeta(G)=G$. (Alternatively, if $T$ is the maximal periodic normal subgroup of $G$, then $T$ is a $\pi^{\prime}$-group, so $T \leq \zeta(G)$ and $\zeta(G / T)$ is isolated in $G / T$ by [6] 2.3.9i); thus again $\zeta(G)=G$.)

The Proof of Theorem $D$. Let $X \leq Y$ be finitely generated subgroups of $G$. Then $X / \zeta(X)$ is a finite $\pi$-group, so by Theorem $\mathrm{C}$ there exists a finite normal $\pi$-subgroup $L_{X}$ of $G$ with $X / L_{X}$ hypercentral and hence nilpotent. Clearly we may choose $L_{X}$ so that $X / L_{X}$ is $\pi$-torsion-free. Then $L_{X}=X \cap L_{Y}$. Set $L=\cup_{X} L_{X}$. Then $L$ is a locally finite, normal $\pi$-subgroup of $G$ with $G / L \pi$-torsion-free and locally nilpotent. By the lemma above $G / L$ is hypercentral.

Example 1 If $G / \zeta(G)$ is polycyclic, there is no need for $G$ to be (polycyclic-by-finite)by-hypercentral.

Let $A$ be a divisible abelian 2-group of rank 2 . Then $A u t A \cong G L\left(2, \mathbb{Z}_{2}\right)$. Let $H=\langle x, h\rangle \leq G L\left(2, \mathbb{Z}_{2}\right)$; here $x \neq 1$ permutes the standard basis of $\left(\mathbb{Z}_{2}\right)^{(2)}$ and $h=\operatorname{diag}\left(k, k^{-1}\right)$ where $k \in 1+2 \mathbb{Z}_{2} \leq \mathbb{Z}_{2}$ has infinite order. Set $A_{i}=\{a \in A$ : $\left.|a| \leq 2^{i}\right\}$ for $i=0,1,2, \ldots$. Then $\left[A_{i}, h\right] \leq A_{i-1}$ for all $i>0$; also $A_{i}^{x}=A_{i}$ and $\left[A_{i}, 2 x\right] \leq A_{i-1}$. Further $H$ is infinite dihedral, so $\zeta_{1}(H)=\langle 1\rangle$.

Let $G=H A$ be the split extension of $A$ by $H$. Then $\zeta(G)=A$ and $G / \zeta(G) \cong H$ is polycyclic. Suppose $T$ is any polycyclic-by-finite normal subgroup of $G$. Then 
$T \cap A \leq A_{i}$ for some $i$. If $m$ is a positive integer with $h^{m} \in T$, then $h^{m}$ stabilizes the series $\langle 1\rangle<A_{1}<A_{2}<\cdots<A_{i}<A$ and hence $h^{m n}$ centralizes $A$ for some $n \geq 1$ (e.g. [11] 1.21), contradicting $h$ of infinite order. Consequently $H \cap T=\langle 1\rangle$ and so $G / T$ cannot be hypercentral.

The Proof of Theorem $F$. Define $K$ by $K / A=(H / A) \cap \zeta(G / A)$. We induct on the exponent $e$ of $A$. Suppose first that $e=p$, a prime and that $\zeta(G)=\langle 1\rangle$. If $K>A$, then there exists $k \in K \backslash A$ with $k A \in \zeta_{1}(G / A)$. Then $V=\langle k\rangle A$ is abelian and normal in $G$ and clearly $\left[v^{p}, g\right]=[v, g]^{p}=1$ for all $v$ in $V$ and $g$ in $G$. Also $V \leq H$, so $G / C_{G}(V)$ is an image of $G / C_{G}(H)$ and hence is nilpotent. It follows that $V \cap \zeta_{1}(G) \neq\langle 1\rangle$, either because $V^{p} \neq\langle 1\rangle$ or by the Remark above, contradicting the assumption that $\zeta(G)=\langle 1\rangle$. Thus in this case $K=A$. Applying this to $G / \zeta(G)$ yields that if $A$ is elementary abelian, then

$$
K / A \leq(H / A) \cap A . \zeta(G) / A=A(H \cap \zeta(G)) / A \leq K / A .
$$

Now suppose that $p$ is just some prime dividing $e$ and set $B=A^{p}$. By the case above

$$
(H / A) \cap \zeta(G / A)=(A / B)((H / B) \cap \zeta(G / B)) /(A / B) .
$$

Also by induction on $e$ we have $(H / B) \cap \zeta(G / B)=B(H \cap \zeta(G)) / B$. Therefore

$$
(H / A) \cap \zeta(G / A)=(A / B)(B(H \cap \zeta(G)) / B) /(A / B)=A(H \cap \zeta(G)) / A .
$$

The proof is complete.

Let $L$ be a finite group of order $d$. Then any series of subgroups of $L$ has length at most $h(d)$, the minimal number of generators of $L$ is at most $h(d)$ and Aut $L$ has order at most $d^{h(d)}$. For example, appying this to Theorem E yields that $(G: \zeta(G))$ is at most $d^{h(d)+1}$.

Assume $k \geq 0$ and $d>1$ are integers and suppose $G$ is a group with $L=\gamma^{k+1} G$ of order $d$. Then from Theorem 2 of [3] we have $\left(G: \zeta_{2 k}(G)\right) \leq d^{s}$, where $s=$ $r^{k}+h(d)$ and $r$ is the rank of Aut L. Note that $r$ is bounded by a function of $d$ only; for example $r \leq h(d)^{2}$. Also $\zeta(G)=\zeta_{k+e(d)}(G)$, see Remarks above; consequently $\left(G: \zeta_{k+e(k)}(G)\right) \leq d^{h(d)+1}$. Thus if $k \geq e(d)$, then $\left(G: \zeta_{2 k}(G)\right) \leq d^{h(d)+1}$ and if $k<e(d)$, then $\left(G: \zeta_{2 k}(G)\right) \leq d^{s}$ for $s=r^{k}+h(d) \leq r^{e(d)}+h(d) \leq h(d)^{2 . e(d)}+$ $h(d)=u(d)$ say. We have proved the following (cf. [1] Corollary $\mathrm{A}^{\prime}$ ). If $\left|\gamma^{k+1} G\right|=d$, then $\left(G: \zeta_{2 k}(G)\right) \leq d^{u(d)}$ for $u$ as above, a function of $d$ only.

Unlike the previous case we need not have that $\left(G: \zeta_{2 k}(G)\right)$ divides a power of $d$, for if $G=\operatorname{Sym}(3)$ and $k=1$, then $d=3$ and $\left(G: \zeta_{2 k}(G)\right)=6$.

For the analogues of Theorem $\mathrm{E}$ the results are negative.

Example 2 If $G$ is (infinite cyclic)-by-hypercentral, then $G / \zeta(G)$ need not be polycyclic-by-finite.

Let $A=\mathbb{Z}, B=\mathbb{Z}[1 / 2], g$ the automorphism $b \mapsto-b$ of $B$ and $G$ the split extension $\langle g\rangle B$. Then $A$ is infinite cyclic and normal in $G$ and $G / A$ is hypercentral, being an infinite locally dihedral 2-group. Finally if $x \in G \backslash B$, then $x$ acts fixed-point freely on $B$, so $\langle 1\rangle=\zeta_{1}(G)=\zeta(G)$. Clearly $G$ is not polycyclic-by-finite. 
Example 3 If $G$ is (locally finite)-by-hypercentral, then $G / \zeta(G)$ need not be periodic.

Let $G$ be the wreath product of a cyclic group of prime order $p$ by an infinite cyclic group. Then $G^{\prime}$ is an elementary abelian $p$-group and yet $\zeta(G)=\zeta_{1}(G)=\langle 1\rangle$.

Example 4 If $G$ is Chernikov-by-hypercentral, then $G / \zeta(G)$ need not be Chernikov or even periodic.

Let $G$ be the split extension of the Prüfer $p$-group $P$ for the odd prime $p$ by the infinite cyclic group $\langle a b\rangle$, where $a$ is the inversion automorphism of $P$ and $b$ is an automorphism of $P$ of infinite order that stabilizes the (only) composition series of $P$. Then $G^{\prime}=P$ and so is Chernikov, but $\zeta(G)=\langle 1\rangle$, so $G / \zeta(G)$ is not even periodic.

Open Access This article is distributed under the terms of the Creative Commons Attribution 4.0 International License (http://creativecommons.org/licenses/by/4.0/), which permits unrestricted use, distribution, and reproduction in any medium, provided you give appropriate credit to the original author(s) and the source, provide a link to the Creative Commons license, and indicate if changes were made.

\section{References}

1. Casolo, C., Dardano, U., Rinauro, S.: Variants of theorems of Baer and Hall on finite-by-hypercentral groups. J. Algebra 452, 279-287 (2016)

2. de Falco, M., de Giovanni, F., Musella, C., Sysak, Y.P.: On the upper central series of infinite groups. Proc. Am. Math. Soc. 139, 385-389 (2011)

3. Hall, P.: Finite-by-nilpotent groups. Proc. Camb. Philos. Soc. 52, 611-616 (1956)

4. Hall, P.: Nilpotent Groups. Queen Mary College Mathematical Notes, London (1969)

5. Kurdachenko, L., Otal, J., Subbotin, I.Y.: On a generalization of Baer's theorem. Proc. Am. Math. Soc. 141, 2597-2602 (2013)

6. Lennox, J.C., Robinson, D.J.S.: The Theory of Infinite Soluble Groups. Clarendon Press, Oxford (2004)

7. Robinson, D.J.S.: Finiteness Conditions and Generalized Soluble Groups, vol. 1. Springer, Berlin (1972)

8. Robinson, D.J.S.: A Course in the Theory of Groups. Springer, Berlin (1982)

9. Shirvani, M., Wehrfritz, B.A.F.: Skew Linear Groups. Cambridge University Press, Cambridge (1986). (re-issued (2008))

10. Wehrfritz, B.A.F.: Infinite Linear Groups. Springer, Berlin (1973)

11. Wehrfritz, B.A.F.: Group and Ring Theoretic Properties of Polycyclic Groups. Springer, London (2009)

12. Wehrfritz, B.A.F.: Schur's theorem and Wiegold's bound (preprint)

13. Wiegold, J.: Multiplicators and groups with finite central factor-groups. Math. Z. 89, 345-347 (1965) 